\title{
Annex I: List of Research Participants
}

About the following table:

- There were about ten more interlocutors who are not listed here but with whom informal conversations or interviews were held.

- Names changed for reasons of anonymity.

- Types of residence status in Switzerland:

o $\quad B=$ Temporary permit of residence (includes a work permit, but additional administrational effort is required by employers to employ persons with a $B$ permit; it usually requires annual renewal)

o $\quad$ C = Permanent permit of residence (includes a work permit; usually issued after five or ten years of residence in Switzerland)

o $\quad F=$ Provisionally accepted refugee

o $\quad \mathrm{L}=$ Kurzaufenthaltsbewilligung (short term permit)

o $\quad \mathrm{N}=$ Asylum claimant (may reside in Switzerland for the duration of the asylum application procedure)

- Exact professions and in some cases the country are not indicated for reasons of anonymity. Professions are sometimes mentioned in the main text.

- $\quad$ e.P. = eingetragene Partnerschaft (registered partnership) 


\begin{tabular}{|c|c|c|c|c|c|c|c|c|c|c|c|c|}
\hline 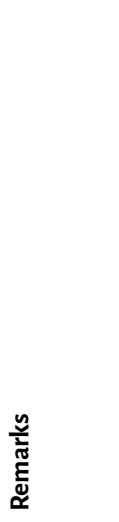 & 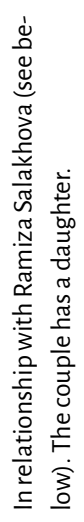 & & 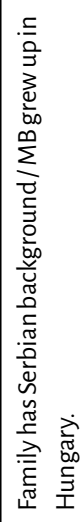 & & 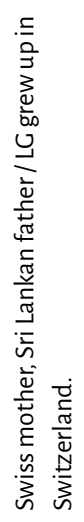 & & 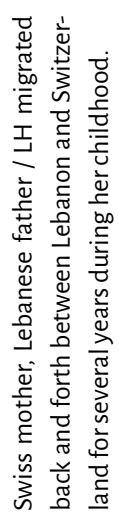 & & & & & 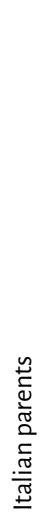 \\
\hline 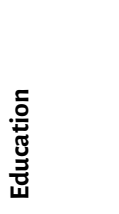 & 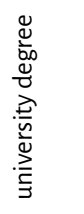 & 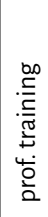 & 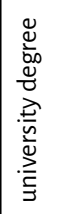 & 1 & 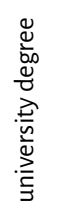 & 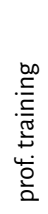 & 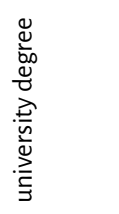 & 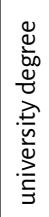 & 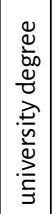 & 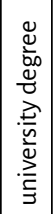 & 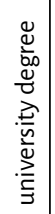 & 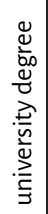 \\
\hline 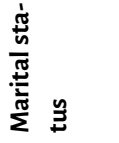 & 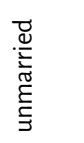 & 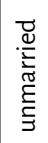 & 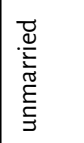 & 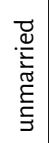 & 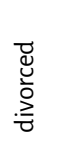 & 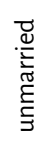 & 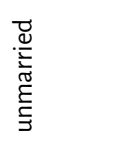 & 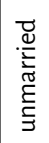 & ن & $\stackrel{\circ}{j}$ & 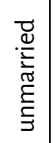 & نं \\
\hline 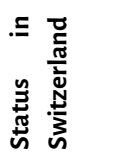 & $z$ & $\infty$ & $\infty$ & 1 & 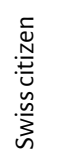 & 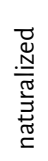 & 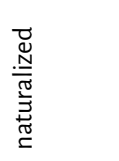 & $\infty$ & 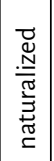 & $\infty$ & $\infty$ & 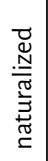 \\
\hline 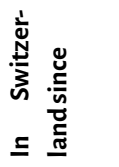 & 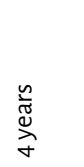 & 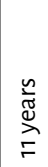 & 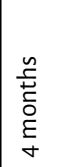 & 1 & 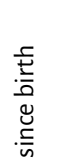 & 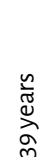 & $\begin{array}{l}\stackrel{n}{\pi} \\
\stackrel{\infty}{\infty} \\
\stackrel{\infty}{N}\end{array}$ & 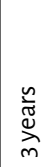 & 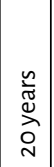 & 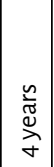 & 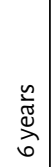 & 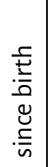 \\
\hline 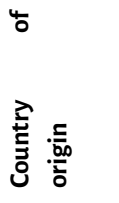 & 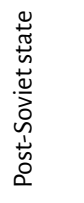 & 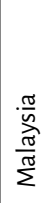 & 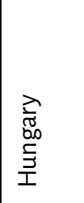 & 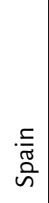 & 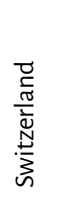 & $\begin{array}{l}\frac{5}{\pi} \\
\text { ñ }\end{array}$ & 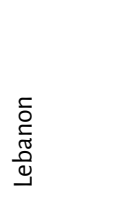 & $\begin{array}{l}\stackrel{\Xi}{\Xi} \\
\text { பँ }\end{array}$ & $\begin{array}{l}\overline{\bar{N}} \\
\overline{\mathscr{D}} \\
\bar{D}\end{array}$ & 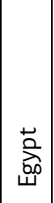 & $\begin{array}{l}\frac{\pi}{0} \\
\frac{.0}{0} \\
\frac{0}{0}\end{array}$ & 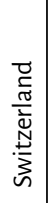 \\
\hline 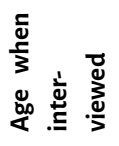 & $\stackrel{\infty}{m}$ & $\tilde{m}$ & $\tilde{m}$ & 1 & $\stackrel{\infty}{m}$ & $\forall$ & बे & $\bar{m}$ & $\dddot{q}$ & $\bar{m}$ & $\tilde{m}$ & 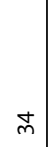 \\
\hline 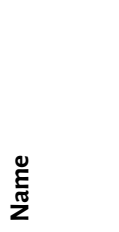 & 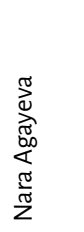 & 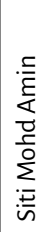 & 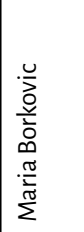 & 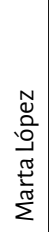 & 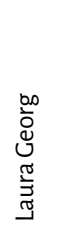 & 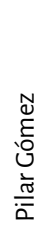 & 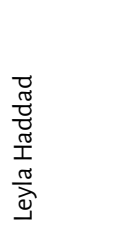 & 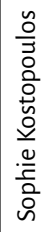 & 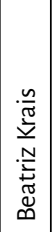 & 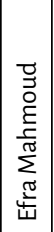 & 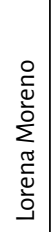 & 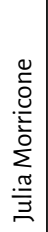 \\
\hline
\end{tabular}




\begin{tabular}{|c|c|c|c|c|c|c|c|c|c|c|}
\hline 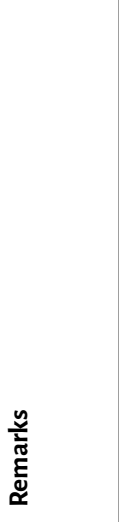 & 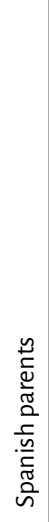 & 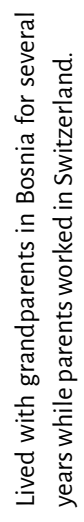 & & & 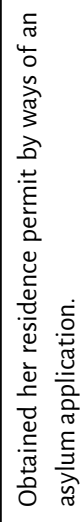 & & & 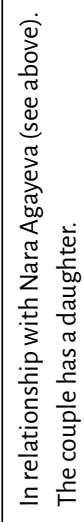 & & 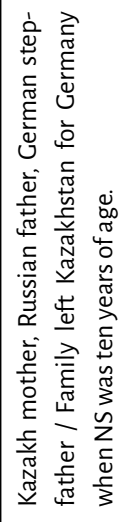 \\
\hline 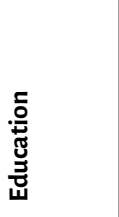 & 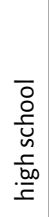 & 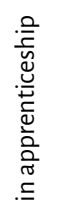 & 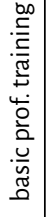 & 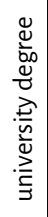 & 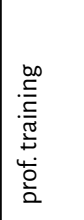 & 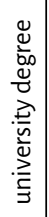 & 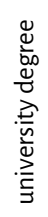 & 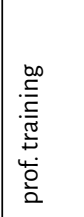 & 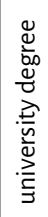 & 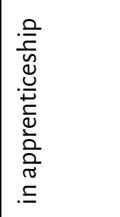 \\
\hline 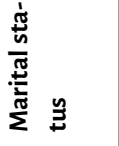 & ن & 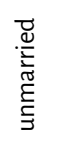 & 1 & 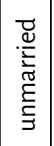 & 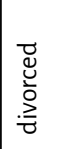 & 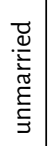 & نे & 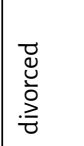 & 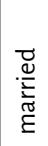 & 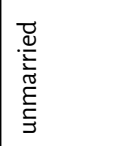 \\
\hline 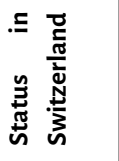 & $u$ & $u$ & 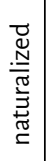 & 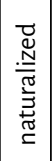 & $u$ & $\infty$ & $\infty$ & $\frac{u}{z}$ & $\cup$ & - \\
\hline 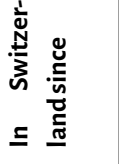 & 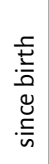 & 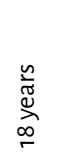 & 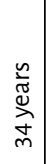 & 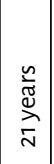 & 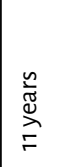 & 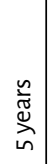 & 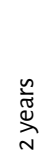 & 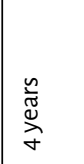 & 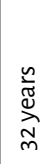 & $\begin{array}{l}\tilde{n} \\
\underline{c} \\
\vdots \\
\vdots \\
\xi \\
\sigma\end{array}$ \\
\hline 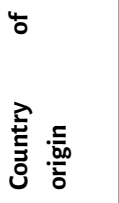 & 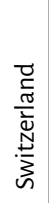 & 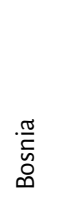 & 竞 & 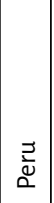 & $\stackrel{\pi}{3}$ & 좊 & 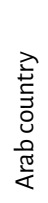 & 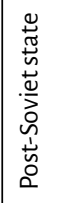 & $\begin{array}{l}\text { శ్ } \\
\text { న్ } \\
\text { న్ }\end{array}$ & 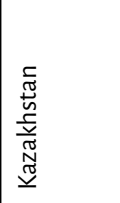 \\
\hline 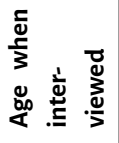 & N & $\stackrel{\infty}{N}$ & it & $\stackrel{\infty}{m}$ & 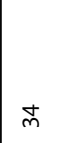 & $\tilde{m}$ & $\hat{m}$ & $\mathcal{F}$ & 8 & ㅇ \\
\hline 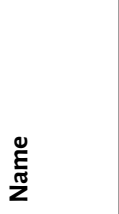 & 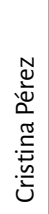 & 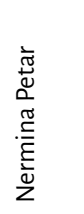 & 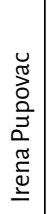 & 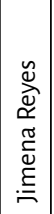 & 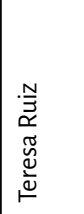 & 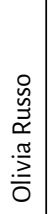 & 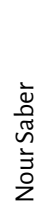 & 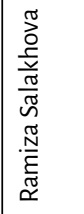 & 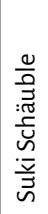 & 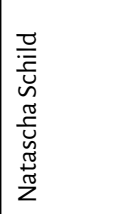 \\
\hline
\end{tabular}




\begin{tabular}{|c|c|c|c|c|c|c|}
\hline 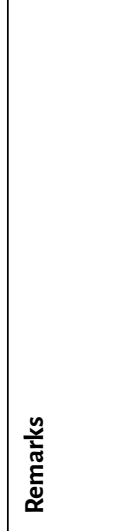 & & & 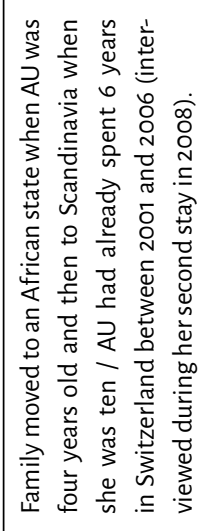 & 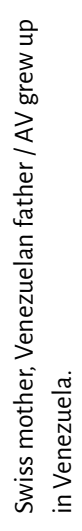 & & 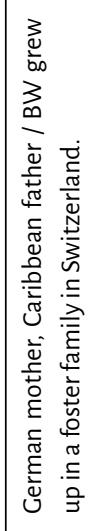 \\
\hline 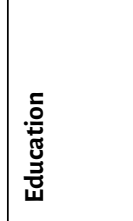 & 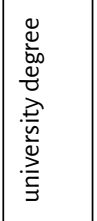 & 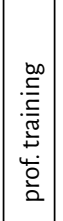 & 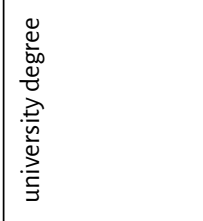 & 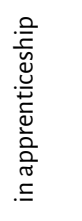 & 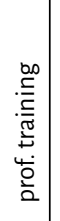 & 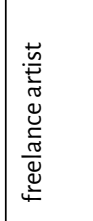 \\
\hline 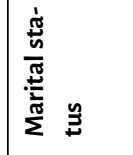 & 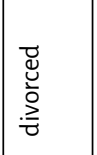 & 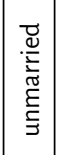 & ن: & ○.ं & $\begin{array}{l}\overrightarrow{\widetilde{U}} \\
\stackrel{0}{0} \\
\stackrel{2}{0}\end{array}$ & 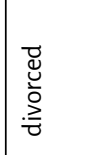 \\
\hline 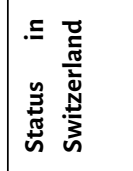 & $\infty$ & $u$ & $\infty$ & 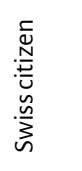 & $u$ & 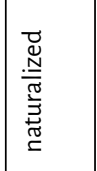 \\
\hline 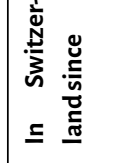 & 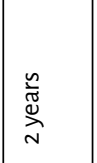 & $\begin{array}{l}\stackrel{n}{\tilde{w}} \\
\stackrel{\tilde{w}}{m} \\
\stackrel{m}{q}\end{array}$ & 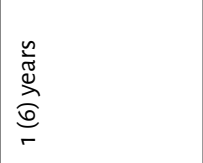 & 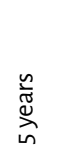 & 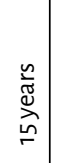 & 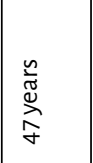 \\
\hline 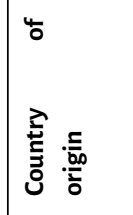 & 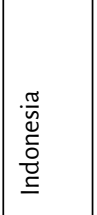 & 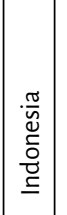 & 胥 & 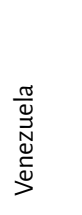 & 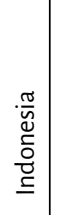 & 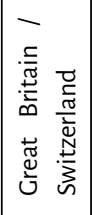 \\
\hline 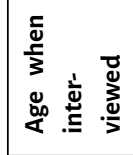 & $\stackrel{\sim}{m}$ & $\bar{m}$ & $\stackrel{+}{m}$ & 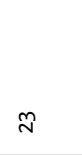 & $\stackrel{\varphi}{m}$ & $\stackrel{\infty}{\sigma}$ \\
\hline 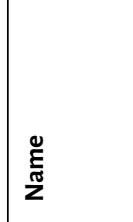 & 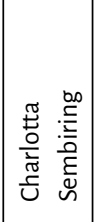 & 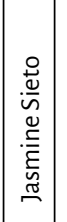 & 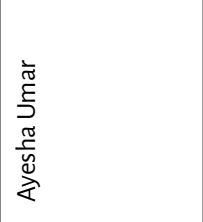 & 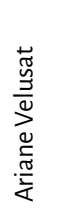 & 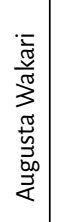 & 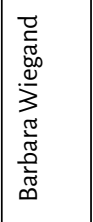 \\
\hline
\end{tabular}

